\title{
Determination of soil heterogeneity by precision farming methods
}

\author{
N. I. Adamchuk-Chala ${ }^{1,2 *}$, V. O. Yatsenko ${ }^{3}$, M. M. Baranovskij ${ }^{2}$, J. V. Bojko ${ }^{4}$, \\ V. I. Adamchuk ${ }^{5}$ \\ ${ }^{1}$ Insitute of Agroecology NAAS of Ukraine, Metrologichna str., 12, 03143, Kyiv, Ukraine \\ ${ }^{2}$ National Aviation University, 03680, av. Guzar, 1, Kyiv \\ ${ }^{3}$ Kyiv National University named Taras Shevchenko. 03680, av. Glushkova, 4d \\ ${ }^{4}$ Agrilab 17500, Chernigivskij reg., Pryluky, a/b 51 \\ ${ }^{5}$ McGill University, 21, 111 Lakeshore Road, PO Box 127, Ste-Anne-de-Bellevue, QC, H9X 3V9, Montreal, \\ Canada \\ Corresponding author E-mail: nadiaadamchuk@gmail.com
}

\section{Received: 17.11.2020. Accepted: 17.12.2020}

\begin{abstract}
The paper presents the results of optimization of crop management in specific areas of the field while taking into account spatial and temporal changes in soil conditions. The aim of the study was to identify soil microbial groups that affect the production cycle with different tillage and the use of different techniques for measuring the biological activity of soil and yield. Main results of Experiment 1: Dilution of $1 / 100000$ of nitrogen fertilizers applied to post-harvest residues provided the highest reproducibility of measurements for the largest number of microorganisms of different ecological and trophic groups. Differences in the mean values of the six repeated measurements on the two repeated samples can be considered significant. Main results of Experiment 2: The addition of CAS-32 in early October did not lead to significant changes in microbiological activity in early November. However, there was a significant difference in microbiological activity for different samples depending on their location and time of the study.
\end{abstract}

Keywords: soil heterogeneity; agriculture; electro physical methods

\section{Introduction}

To assure sustainable productivity of agricultural fields, it is important to balance supply of essential nutrients according to local soil and climatic conditions. Soil amendments are commonly used to assure sufficient availability of key nutrients through the growing season. The types and amounts of these amendments have been varied according to management practices and soil nutrients content. However, both carbon and nitrogen cycles that affect soils fertility depend in microbiological activity. However, little research has been published on direct investigation of spatial variability in the quantity of key groups of microorganisms through the growing season. In addition, certain conservation treatments are aimed at stimulating activity of microorganisms, and reports on quantitative assessment of direct impact of such treatments have been limited

Since the beginning of the 90 s of the last century, during the massive spread of information technology, farmers for the first time began to separate agricultural precision from the general development of agriculture. The formation of agricultural precision (AP) technologies was facilitated by the latest technical advances in the development of society, such as creation of reliable and highly productive means of agricultural mechanization; rapid spread of electronics and information technology, the availability of global positioning systems. AP technologies, which are actively used in the world, provide an opportunity to match the quantity and quality of agricultural materials. They are always used with the conditions of individual areas of the field.

Nowadays, global positioning devices (GPS), remote sensing methods and geographic information systems (GIS) are widely used in various industries. In agriculture, these information technologies provide accurate and automated collection, analysis and storage of qualitative and quantitative information about soils. The main components of the vehicle system include: global positioning systems (GSP), geographic information systems (GIS), technologies of variable rates of application (ZNV) of technological materials (TM), crop mapping tools, remote monitoring systems: systems and methods for measuring plant parameters and soil properties. Geographic Information Systems (GIS) is a set of software and techniques for accumulation, processing, storing and interpretation of geographically linked data. The main task of these software products is the implementation of developed and improved algorithms for filtering and analyzing visual data and they are also used to organize the information flows.

The software provides the ability to visualize the crop map (with a considerable level of error) and its transformation into a map of the specified variable rates of technological materials application. On the other hand, more reliable results can be expected if a number of filtered crop cartograms from one field is combined with cartograms characterizing soil properties or with aerial or space images in determining statistically (cluster classification) zones of differential management.

These new technologies of variable norms of introduction and technological materials, calculation of costs for accumulation and data processing will not lead to improvement of economic indicators of production until there is a reasonable change of management strategy for agrobiological potential of the field. Such changes are most effective in the introduction of AP methods to calculate the rates of application of technical materials (TM) (seeds, fertilizers, limestone, and chemicals). 
With an automatic control system, the control unit sets the application rate that corresponds to the value of the pre-compiled map or taking into account the signal from the sensor that determines the parameters of the soil or plants in real time. Thus, the control device assumes the functions of changing the rates of application of TM, while the operator performs traditional work.

In advanced countries, electrophysical methods are used for soil surveys. In North America and Europe, the use of soil conductivity maps (SOEs) reduces survey costs and provides more objective and representative information on changes in soil properties in space compared to surveys used only on classical methods (Shiratsuchi, 2009). Leading agro-consulting firms in many countries use KEG as one of the elements to study the parameters of soil heterogeneity.

Electrical conductivity of the soil is the ability of its constituent particles to conduct electric current, inverse to the electrical resistance. It is expressed in units of millimeters (per $\mathrm{mSm} / \mathrm{m}$ ). Electrical conductivity combines many soil properties: soil moisture, particle size distribution, salinity, content of exchangeable cations of calcium, magnesium, etc. They influence the architecture of the soil microbiota, as well as crop yields.

Aim of investigation - assessing effects of soil additives on microbiological activity in chernozem soils.

\section{Materials and Methods}

Soil mapping was performed using an off-road car combined with an on-board computer with parallel driving technology with a GPS receiver, a Veris $3100 \mathrm{EP}$ device and a trailer unit with disks (with electrodes placed in the disks).

Experiment 1: In October, six soil samples have been collected from three different locations in an agricultural field (specifics to be added as needed). The two replicated samples have been obtained from specific field areas representing one of three treatment practices pursued under similar agro-climatic conditions. The areas were defined according to the application maps. Since the capability to conduct this experiment emerged after the treatments, quality of samples should be treated as is that do not possess experimental design requirements. Samples 1 and 6 represented soil with corn residue with 5 L/ha of Effect Bio and 10 L/ha UAN 32 applied. Areas represented by samples 2 and 3 were cultivated after winter wheat with ammonia nitrate application. Finally, samples 4 and 5 represent soil with corn residue with 100 L/ha of UAN 32 applied. Microbiology_2010.xls EXCEL file contains the data obtained.

Experiment 2: In this case, a single corn field has been sampled two times (before UAN application for mineralization of plant residues and one month after that. Fig. 3 illustrates the yield map with 19 samples (samples 1-12 have been analyzed for microbiological activity) and Fig. 4 illustrates UAN-32 application rate and a similar sampling scheme. Microbiology_2011.xls EXCEL file contains the data obtained. Figure 5 shows change of microbiological measurements between two tests.

Agronomic parameters for these samples are presented in Table 1

Table 1. Sample characteristics.

\begin{tabular}{llllll}
\hline $\begin{array}{l}\text { Sample } \\
\text { ID }\end{array}$ & Treatment & $\mathrm{pH}, \mathrm{KCl}$ & $\begin{array}{c}\mathrm{NH4}, \\
\mathrm{mg} / \mathrm{kg}\end{array}$ & $\begin{array}{c}\mathrm{NO3}, \\
\mathrm{mg} / \mathrm{kg}\end{array}$ & $\begin{array}{c}\text { Mineral N, } \\
\mathrm{mg} / \mathrm{kg}\end{array}$ \\
\hline 1 & $\begin{array}{l}\text { Corn residue + Effect Bio (5 L/ha) + UAN-32 } \\
\text { (10L/ha) }\end{array}$ & 6.0 & 4.2 & 17.2 & 21.4 \\
6 & Corn residue + Effect Bio (5 L/ha) + UAN-32 & 6.0 & 5.4 & 13.4 & 18.8 \\
2 & (10 L/ha) & 6.2 & 5.4 & 12.6 & 18.0 \\
3 & Wheat residue + aqua ammonia & 5.6 & 6.2 & 15.5 & 21.7 \\
4 & Wheat residue + aqua ammonia & 5.5 & 8.3 & 26.6 & 34.9 \\
5 & Corn residue + UAN-32 (100 L/ha) & 5.3 & 8.9 & 28.2 & 37.1 \\
\hline
\end{tabular}

Ecological and trophycal microorganisms were identified according to Vekirchyk (2006).

\section{Results and discussion}

Fig. 1 illustrates change of reproducibility (RMSE) values influenced by dilution of prepared samples and Fig. 2 shows the results of microbiological tests.

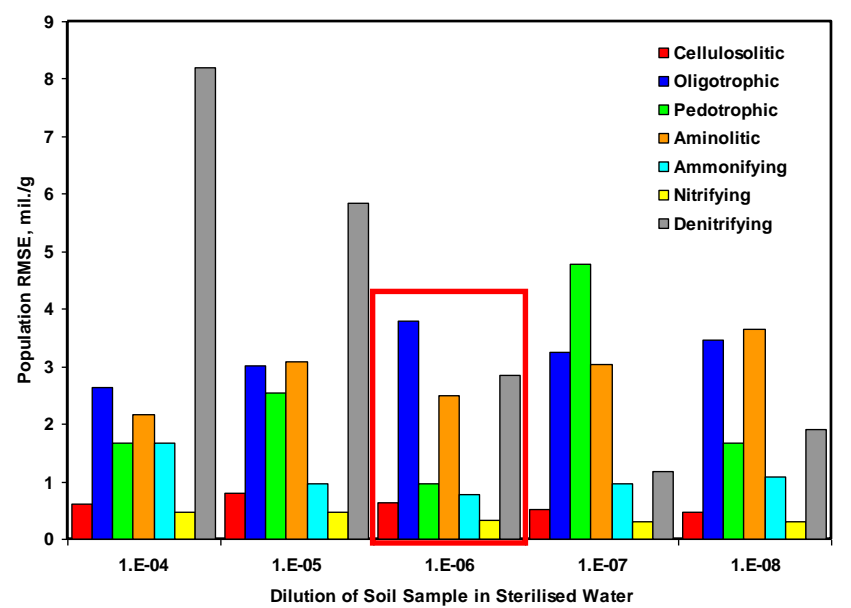

Fig. 1. Reproducibility of measurements affected by sample solution. 

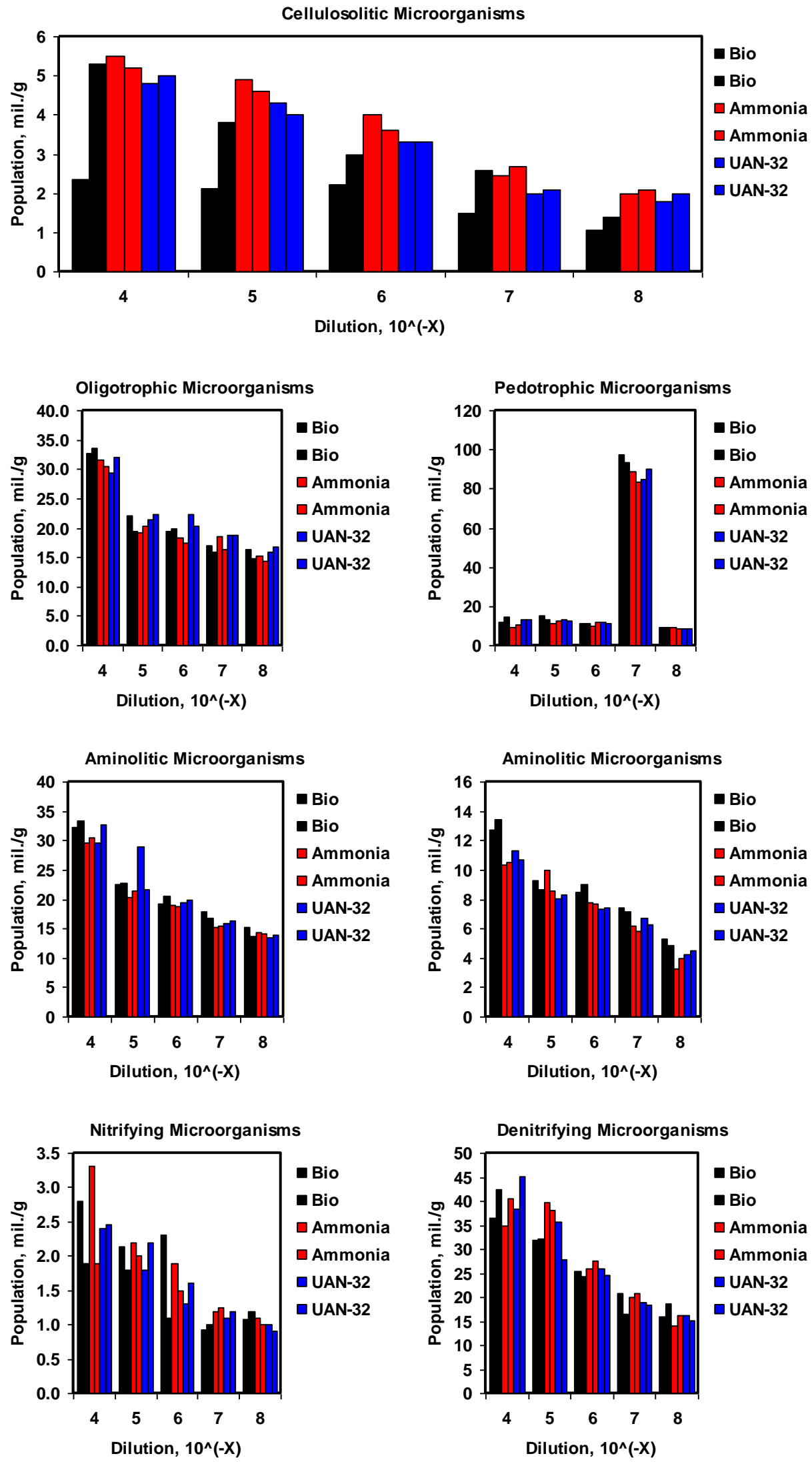

Fig. 2. Sample average test results.

The main source of nitrates in soils and in the biosphere is the activity of microorganisms. The microbiological transformation of nitrogen combinations into nitrates plays an extremely important role in the nitrogen cycle in nature and directly affects the intensity of other processes of the nitrogen cycle. Thus, particularly, the high content of nitrates in soils leads to the development of denitrification processes, as well as limits the activity of the nitrogen fixation process.

Due to the fact that the optimal conditions for the process of nitrification and cultivation of most crops are quite similar, and nitrates are the optimal form of nitrogen for assimilation by plants, for a long time nitrifying activity was considered to be one of the objective indicators of soil fertility. However, the development of research on this issue has led to the understanding that the excessive formation of nitrates in the soils of agrocenoses is the main cause of many environmental problems (Iutinskaya, 2006). 
Nitrates are being easily washed out from soil horizons into groundwater. They are also a direct substrate for denitrifying microorganisms. As a result, they enter the atmosphere in the form of $\mathrm{N}_{2} \mathrm{O}$ and $\mathrm{N}_{2}$.
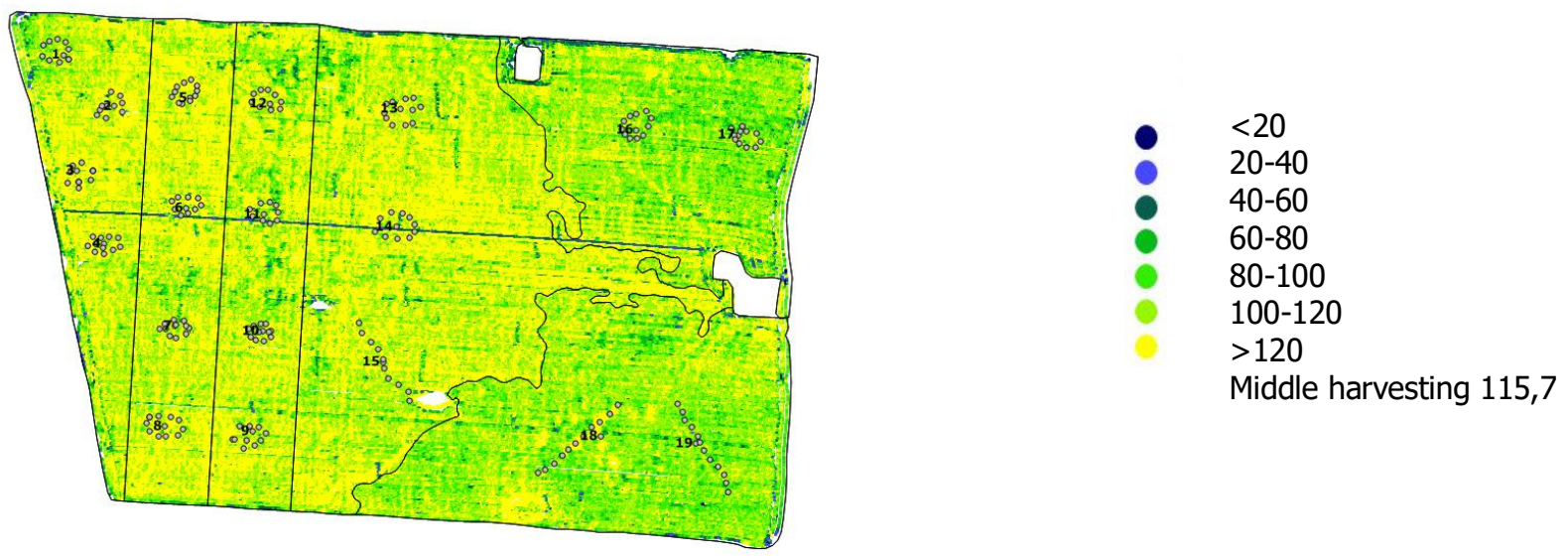

Fig. 3. Corn yield and September 28 sampling. Fild $11 \mathrm{H}$ Squer 312 ha, Corn yield, centner/ha.

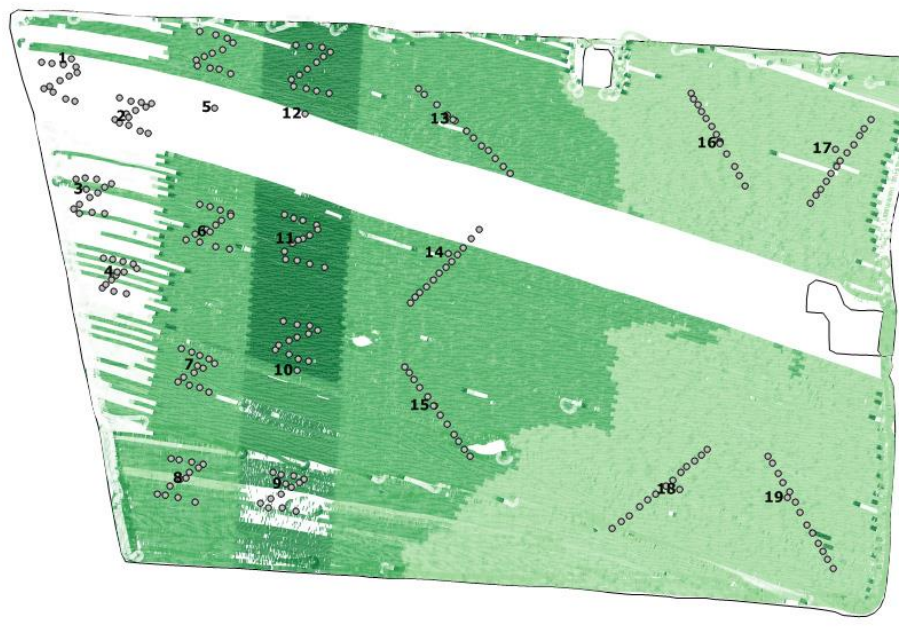

0

100

120

230

Fig. 4. UAN-32 application map and November 9 sampling. Fild $11 \mathrm{H}$ Squer 312 ha, soil with corn residue with UAN-32 applied, L/ha

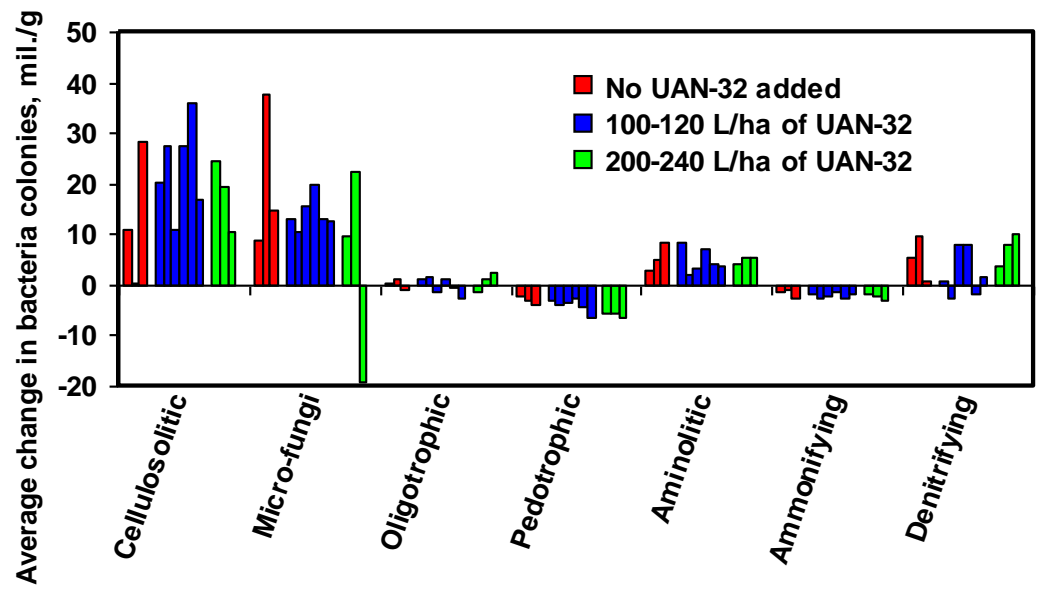

Fig. 5. Average change on microbiological measurements between two tests.

Indicators of general biological activity of soil are widely used in microbiological and agronomic practice as a reflection of total microbiological activity. However, it should be remembered that the growth of total biological activity may not always be a measure of positive changes in microbiological processes in the soil. Thus, in agrocenoses, it is necessary to achieve not the maximum 
activity of microbiological processes in the soil, but to bring their intensity to the optimal level for specific conditions. In this regard, it should be noted some methodological limitations and contradictions of existing in soil microbiology and soil science approaches to determining the biological activity of soil, for example, the intensity of $\mathrm{CO}_{2}$ production. In the conditions of natural ecosystems, where the cycle of biophilic elements is mainly the result of microbiological activity, these indicators can be objective; there is no such dependence in cultivated soils.

Earlier it was seen that the reserves of potential soil fertility are almost limitless and it should be actively transformed into mineral compounds for plant nutrition. As it turned out, this view can only be correct for natural ecosystems. In agroecosystems, with unbalanced processes of synthesis and mineralization of humus, its active using, as can be stated today, can do more harm than good. The potential fertility of soils for agricultural use is actively declining and in some agrocenoses has reached a critical level. Another example of incorrect assessment of the biological condition of soils in terms of overall biological activity is the conduct of appropriate tests using deliberately high doses of nitrogen fertilizers. In this case, the overall biological activity will be high, but mainly, due to the activity of denitrifying microorganisms. To interpret the growth of biological activity as a benefit for the farmer in this case would be a mistake. Ecologically safe methods of nitrogen nitrification control in agrocenoses will increase microbial biomass reserves, which can be achieved by adding organic matter, using slow-acting ammonium fertilizers, etc. A number of microbiological processes involving three ecological and trophic groups of soil microorganisms detected on selective media. Ammonifying microorganisms are involved in the decomposition of nitrogen-containing compounds with the release of ammonia (Volkogon, et al. 2010). The decomposition of simple proteins occurs with the participation of extracellular and intracellular enzymes (protease, peptidase) in the following sequence:

protein $\rightarrow$ peptones $\rightarrow$ peptides $\rightarrow$ amino acids

The quantitative presence and activity of the soil microbiota directly depends on the ambient temperature and the level of moisture in the wet soil while maintaining a stable ratio of carbon to nitrogen (C:N) (Vekirchyk, 2006; Schlegel, 1987). In such a way, biological nitrogen is returned to the soil in two natural ways - by decomposition of cellulose and associative nitrogen fixation. This is the way back to the soil of carbon dioxide (up to $25 \%$ of the total mass of straw) used by plants for photosynthesis. When water is combined with carbon dioxide, it turns into carbonic acid, which helps to convert a certain number of soil elements into a soluble form. Nowadays, it is known that among soil microorganisms there are two groups of nitrifiers: the first oxidizes ammonium to nitrite, and the second - nitrite to nitrate. Representatives of the first phase of nitrification receive energy for biosynthetic processes during the oxidation of ammonia to nitrite, and the second - respectively, during the oxidation of nitrite to nitrate. Bacteria use $\mathrm{CO} 2$ as a source of carbon.

At the same time the plant will be able to limit the development of nitrifiers only in the absence of ammonium deficiency. At introduction of considerable quantities of ammonium fertilizers in rhizosphere soil it is possible to expect considerable intensification of process of heterotrophic nitrification (Vekirchyk, 2006). It has long been believed, that denitrification in agrocenoses, even with the additional application of mineral nitrogen can not reach large sizes, because it is an aerobic process. However, it was further proved that due to the presence of aerobic microzones in the soil when applying mineral nitrogen fertilizers, the denitrification process can be extremely active, especially in the rhizosphere of crops, due to the presence of easily digestible organic compounds in the root secretions. That is why modern agrocenoses, which use intensive technologies for growing crops, involving the use of high doses of nitrogen fertilizers, should be considered as the source of the largest gaseous losses of nitrogen compounds.

Today, it has been established that during nitrate respiration, the main final reduced compound may not be N2, as previously thought, but nitrous oxide. Since $\mathrm{N}_{2} \mathrm{O}$ is also actively released during the nitrification process, determining the content of nitrous oxide in the medium, it is probably more specific not to lose nitrogen due to the denitrification process, but to emit nitrous oxide during two closely related processes - nitrification and denitrification.

Currently, nitrification is defined as a biological process of ammonium oxidation by highly specialized hemolithoautotrophic bacteria into nitrites, and then into nitrates, in the case of heterotrophic microorganisms and various organic nitrogen compounds (Umarov, 2007). In order to accelerate the decomposition process, compensatory application of nitrogen fertilizers is used. In our opinion, such an agricultural measure will stimulate the active development of microorganisms that will decompose straw in arable land. Therefore, on the basis of yield maps, we divided the field into zones with the same level of productivity, in which samples are taken to determine the total mass of residues and the ratio of $\mathrm{C}$ : $\mathrm{N}$ in them.

The introduction of compensatory rates of nitrogen fertilizers on arable land, included the following stages:

- computer mapping of yields during harvesting by combines,

- high-quality crushing and scattering of plant rests in the field,

- laboratory determination of carbon and nitrogen content and their ratio in straw (number and activity of the main ecological and trophic groups of soil microorganisms, microstructural characteristics and soil biomass).

- information analysis of data by special software,

- development of application maps-tasks for differentiated application of liquid nitrogen fertilizers in the field,

- creation of modern agricultural machinery equipped with a system for applying the dosed rate of fertilizers.

Changes in the main ecological and trophic groups of microorganisms in soils before and after application of nitrogen fertilizers on crop residues are presented. The increase in the number of cellulosolytic microorganisms depends on the growth of nitrogen content in free form during the decomposition of cellulose and fiber of plant post-harvest residues. Pedotrophs within probable intervals. Since pedotrophic and oligotrophic microorganisms are not directly involved in the transport of nitrogen compounds in the soil, the introduction of ammonia compounds did not affect the content and composition of these groups of soil microorganisms. The change in the content of aminolytic microorganisms may be associated with a shift in soil pH to the alkaline side. Ammonifiers are involved in protein transformation. There is a lot of mineral nitrogen in the studied soil, which is utilized by this group of microorganisms, so their proteolytic activity is not expressed.

Due to the increase in nitrogen fertilizers, which are the substrate for nitrifying bacteria, this group of soil microorganisms is the first to be connected to a source of nitrogen, the conversion of nitrites into nitrate form. The number of denitrifying bacteria had a slight tendency to increase. Further, according to the ratio of the obtained data, using the methods (Iutinskaya, 2006), we calculated coefficients and indices that reflect the elements of the morpho-functional structure of microbial coenoses and reveal the direction of processes occurring in certain habitats.

Thus, the inertia index is the ratio of the number of microorganisms taken into account under a microscope to the number of microorganisms grown on soil agar. In our experiment after treatment with nitrogen fertilizers, this index was much higher than the control and had the maximum value after the introduction of ammonia water.

The pedotrophic index is expressed as the ratio of the number of microorganisms growing on soil agar to the number of 
microorganisms on rich organic media (eg, IPA). It shows the functionality of the structure of the microbial cenosis of the soil. According to our experiment, a high index of pedotrophic was characteristic of soils in the control and treatment with ammonia water. With the use of carbonate-ammonia mixture (CAS), which, unlike ammonia water, is not able to evaporate over time, the index decreased. Thus, the functionality of the structure of the microbial coenosis of the soil during its treatment with nitrogen fertilizers based on CAS was reduced.

The oligotrophic coefficient demonstrates the supply of easily digestible nutrients to the soil, which is expressed by the ratio of the number of microorganisms detected in "poor" environments to their number grown on "rich" nutrient media such as IPA. According to the results of our experiment, the oligotrophic coefficient increased in the soil in the second and third variants of nitrogen fertilizer application and decreased, compared with the control, with the complex application of the Bio and CAS Effect. At the same time, the introduction of CAS into the soil was an order of magnitude less than in the third variant of treatment. Thus, the application of tillage with post-harvest residues with ammonia water $5 \mathrm{I} /$ ha or CAS at a dose of $100 \mathrm{I} /$ ha increases the provision of soil with easily digestible nutrients for biological organisms.

Other trends in the functioning of soil microorganisms are shown by the coefficient of mineralization and immobilization of nitrogen. Nitrogen mineralization and immobilization coefficient is an indicator of the intensity of mineralization and assimilation of nitrogen compounds in the soil, expressed by the ratio of the number of microorganisms growing on the nutrient medium with ammonium nitrogen (starch ammonia agar) to the number of microorganisms detected on the nutrient medium.

When using complex nitrogen fertilizers of the first variant, a high coefficient of mineralization was revealed, which indicates that the processes of nitrogen mineralization and immobilization are actively taking place in this soil. In comparison with the control (virgin soil), the second and third variants of nitrogen fertilizers application showed lower values of this coefficient and indicate a slow process of decomposition-synthesis of organic matter in the soil.

Total costs for the purchase of fertilizers were reduced by UAH 644. per hectare. The savings of fertilizers with differentiated application in comparison with the traditional application technology (with one average rate for the field and the use of reference data on the ratio of $\mathrm{C}$ : $\mathrm{N}$ and the main and by-products), ranged from 4.1 to $42.2 \%$. The most active process of decomposition of post-harvest residues occurs at a ratio of $\mathrm{C}: \mathrm{N}=20-30: 1$, and in straw it is 50-100: 1 . In order to optimize the mineralization conditions and achieve a lower value of the $\mathrm{C}$ : $\mathrm{N}$ ratio, it is necessary to ensure a minimum $\mathrm{C}$ : $\mathrm{N}$ balance by applying nitrogen fertilizers.

Consequently, with a winter wheat grain yield of $4 \mathrm{t} / \mathrm{ha}$, the cost of nitrogen left in the field with straw will average $184 \mathrm{UAH} / \mathrm{ha}$, phosphorus - $120 \mathrm{UAH} / \mathrm{ha}$, potassium - $340 \mathrm{UAH} / \mathrm{ha}$. That is, the total cost of purchasing fertilizers is reduced by 644 hryvnia per hectare. With a corn yield of $7 \mathrm{t} / \mathrm{ha}$, the cost of nitrogen left in the field with straw will average $625 \mathrm{UAH} / \mathrm{ha}$, phosphorus - 180 $\mathrm{UAH} / \mathrm{ha}$, potassium - $884 \mathrm{UAH}$ / ha. Thus, the total cost of purchasing fertilizers is reduced by 1689 hryvnias per hectare. And this is a saving only on the purchase of fertilizers, while there are also savings on transportation, storage and application of fertilizers. In our opinion, the differentiated application of liquid nitrogen fertilizers in the zones selected according to the maps of the distribution of the actual yield in the field contributed to the leveling of crops and obtaining the planned yield.

\section{Conclusion}

In conditions of high cost of mineral fertilizers and the absence or presence of a small amount of organic, one of the effective means of replenishment of organic matter and nutrients in the soil is the use of plant residues as fertilizer, while applying compensatory rates of nitrogen fertilizers. Differentiated application of fertilizers allows up to $40 \%$ to reduce the amount of nitrogen fertilizers and rationally distribute them in the field.

Accordance o results of experiment 1 based on RMSE estimates, 1/100,000 dilution appears to produce the greatest measurement reproducibility for the largest number of bacteria types. Some differences between means of six replicated measurements on two replicated samples can be considered significant.

In experiment 2 were finded the significant difference among microbiological activities for different samples, these differences are dominated by geospatial locations rather than soil treatment. A great presence of temporal inconsistency in values observed, and an increase in nitrification bacteria raises questions on validity and cause of such a big change for all 12 samples. Any hints for existence of such relationships were stronger for September data rather than November, which obviously could not be attributed to the treatments. Therefore, these data suggest that adding UAN-32 in the beginning of October did not produce a remarkable change in microbiological activity in the beginning of November for this agricultural field, as tested.

\section{References}

Iutinskaya, G.O. (2006). Soil microbiology. Aristei.

Schlegel, G. (1987). General microbiology. Moscow. Mir.

Shiratsuchi, L.S., Ferguson, R.B., Adamchuk, V.I., Shanahan, J.F., Slater, G.P. (2009). Integration of ultrasonic and active canopy sensors to estimate the in-season nitrogen content for corn. Proceed. North-Central Extension-Industry Soil Fertility Conf, 18-19 Nov. 2009, DesMoines. Int. Plant Nutrition Inst., DesMoines, Brookings, SD.

Umarov, M.M., Kurakov, A.V., Stepanov, A.A. (2007). Microbiological transformation of nitrogen in the soil. Moscow. Geos.

Vekirchyk, K.M. (2006). Workshop on microbiology. Kiyv. Lybid.

Volkogon, V.V., Nadkernichna, O.V., Tokmakova, L.M., Melnichuk, T.M., Tchaikovsky, L.O. (2010). Experimental soil microbiology. Kiyv. Agrarian Science.

\section{Citation:}

Adamchuk-Chala, N.I., Yatsenko, V.O., Baranovskij, M.M., Bojko, J.V., Adamchuk, V.I. (2020). Determination of soil heterogeneity by precision farming methods. Ukrainian Journal of Ecology, 10(6), $42-47$.

(cc) $\mathrm{EY}$ This work is licensed under a Creative Commons Attribution 4.0. License 